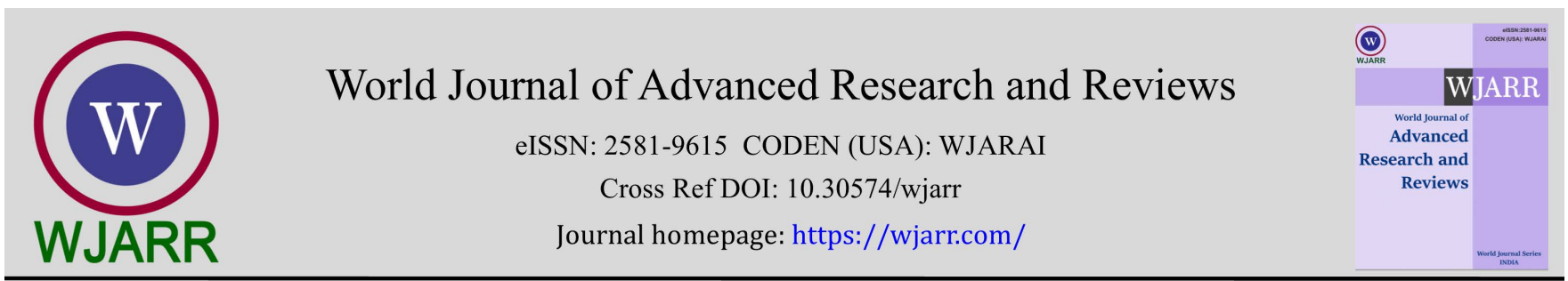

(RESEARCH ARTiClE)

\title{
The effects of gestational age on the composition and predicted function of gut microbiota in neonates and early infants
}

\author{
Kaiyu Pan 1, Lianfang Yu 1, Chengyue Zhang ${ }^{2}$, Jianhua Zhan ${ }^{1}$ and Rongliang Tu ${ }^{3, *}$ \\ ${ }^{1}$ Department of Paediatrics, The First People's Hospital of Xiaoshan District, Hangzhou, Zhejiang, China. \\ 2 Xiangya School of Medicine, Central South University, Changsha, Hunan, China. \\ ${ }^{3}$ Department of Neonatology, Zhejiang Xiaoshan hospital, Hangzhou, Zhejiang, China.
}

World Journal of Advanced Research and Reviews, 2021, 12(02), 567-573

Publication history: Received on 21 October 2021; revised on 24 November 2021; accepted on 26 November 2021

Article DOI: https://doi.org/10.30574/wjarr.2021.12.2.0648

\begin{abstract}
Gut microbiota can influence cell differentiation, metabolism, and immune function and is key for the normal development and future health of early infants. Several factors have been reported to be related to the microbiota composition of neonates, such as gestational age, delivery mode, feeding method, antibiotics consumption, and ethnicity, among others. So we investigated the relationship between gestational age and the composition and predicted function of the gut microbiota of neonates and early infants by sequencing the 16S rRNA gene present in stool samples obtained from 100 prospectively enrolled full-term and preterm newborns. In the 3-day-old neonates samples, the prominent genera in the full-term group were Escherichia-Shigella, Streptococcus, Bifidobacterium, and Bacteroides, while in the preterm group, Staphylococcus, Streptococcus, Escherichia-Shigella and Clostridium were the most abundant genera identified. There were statistical difference between two groups $(P<0.05)$. Moreover, the predominant genera in the full-term group were Bifidobacterium, Lactobacillus, Bacteroides, and Clostridium, whereas the main genera in the preterm group were Escherichia-Shigella, Clostridium, Bifidobacterium and Bacteroides, in stool samples from 30-42day-old infants. We found the $\alpha$-diversity in 3-day-old group was significantly lower than in the 30-42-day-old group whether it's full-term or preterm $(P<0.001)$. Functional inference analysis revealed higher levels of biodegradation and metabolism of carbohydrates, vitamins in the full-term group than in the preterm group, both in neonates and early infants, which may contribute to the stability of the microbiota in the full-term group. There were significant differences in the composition and predicted function of the gut microbiota of early infants due to gestational age. The $16 \mathrm{~S}$ sequencing technology was an effective and reliable tool in the detection of gut microbiota in early infants.
\end{abstract}

Keywords: Full-term; Preterm; Early infants; Gut microbiota; $\alpha$-diversity; Predicted function

\section{Introduction}

The gut microbiota is important for the normal development and future health of early infants, and can greatly impact on metabolism, cell differentiation, and immune function[1] [2] [3]. During the neonatal period, gut microbiota development is influenced from the colonization stage to maturity, by several early-life factors, such as gestational age, delivery and feeding method, and antibiotics consumption, especially in infants and young children [4] [5] [6].These factors will condition the appearance of medical conditions, for example, obesity [7].

Nevertheless, when and how the microbial communities acquire composition and function feature during the early life of a person remains unknow. Recently, the effects of gestational age on the gut microbiota of infants has been subjected to scrutiny. At present, there are few studies about how gestational age affects the composition and predicted function

\footnotetext{
*Corresponding author: Rongliang Tu; E-mail:pky2003@126.com

Department of Neonatology, Zhejiang Xiaoshan hospital, Hangzhou, Zhejiang, China.

Copyright (C) 2021 Author(s) retain the copyright of this article. This article is published under the terms of the Creative Commons Attribution Liscense 4.0.
} 
of the gut microbiota of infants from different ethnicities; however, to the best of our knowledge, Chinese population have not been studied. Currently, a databank of the gut microbiota of Chinese early infants is under construction.

In order to study the effects of gestational age on the gut microbiota of neonates and early infants, 100 newborns were enrolled for sampling during 30-42 days after delivery. Stool sample sequencing analyses of the 16S rRNA gene were performed to study the structure and predicted function of the microbiota of 3- and 30-42-day-old infants.

\section{Material and methods}

\subsection{Volunteers}

We enrolled 100 newborns ( 47 boys and 53 girls), of which 61 were born full-term and 39 preterm. All newborns were Han Chinese ethnicity, born between the $12^{\text {th }}$ of May of 2019 and the $10^{\text {th }}$ of December of 2020, did not receive perinatal antibiotics, and the registered time between premature rupture of membranes and delivery was less than 18 hours. The birth weight of full-term infants was $2.5-4 \mathrm{~kg}$, while that of preterm infants was below $2.5 \mathrm{~kg}$. There were no statistical differences between the two groups regarding gender, delivery mode, and time of premature rupture of membranes. Chromosomal abnormalities, congenital malformations and critical illness with short life expectancy were excluded. The detailed information is presented in Table 1.This project was approved by the Ethics Board of The First People's Hospital of Xiaoshan (Protocol Number: 2019-XS-05). All participants signed the informed consent.

Table 1 Description of 100 participants included in the present study

\begin{tabular}{|l|l|l|l|}
\hline & Gestational age & & \\
\hline Group & $\begin{array}{l}\text { Full-term } \\
\mathbf{n = 6 1}\end{array}$ & $\begin{array}{l}\text { Preterm } \\
\mathbf{n = 3 9}\end{array}$ & P value \\
\hline Sex(\%) & & & 0.892 \\
\hline boys & $29(47.5 \%)$ & $18(46.2 \%)$ & \\
\hline girls & $32(52.5 \%)$ & $21(53.8 \%)$ & \\
\hline Premature rupture of membranes(h) & $3.2 \pm 2.0$ & $3.4 \pm 2.4$ & 0.579 \\
\hline Feeding(\%)3-day-old & & & 0.739 \\
\hline Breast-fed & $28(45.9 \%)$ & $15(38.5 \%)$ & \\
\hline Mixed-fed & $19(31.1 \%)$ & $13(33.3 \%)$ & \\
\hline Fomula-fed & $14(23.0 \%)$ & $11(28.2 \%)$ & \\
\hline Feeding(\%)30-42-day-old & & & 0.975 \\
\hline Breast-fed & $26(42.6 \%)$ & $16(41.0 \%)$ & \\
\hline Mixed-fed & $19(31.1 \%)$ & $12(30.8 \%)$ & \\
\hline Fomula-fed & $16(26.2 \%)$ & $11(28.2 \%)$ & \\
\hline
\end{tabular}

Mean \pm SD for continuous variables: P value was calculated by weighted linear regression model.

$\%$ for categorical variables: P value was calculated by weighted chi-square test.

\subsection{Sample Collection and Processing}

$200 \mathrm{mg}$ of specimens were collected at days 3 and 30-42 after birth by the parents, moved to 2 ml centrifuge tubes, and mixed with $1 \mathrm{ml}$ RNA. All specimens were placed at $4^{\circ} \mathrm{C}$ for $8-12$ hours, then were frozen at $-80^{\circ} \mathrm{C}$. The DNA extracted from the specimens were pooled and sequenced, PCR amplification, fluorescence quantification, and through the construction and sequencing of a MiSeq Library by Bio-science (Hangzhou, Zhejiang).

\subsection{S rRNA Gene Sequencing Analysis}

From the extracted DNA, the hypervariable regions of the $16 \mathrm{~S}$ ribosomal gene were sequenced and an interactive cloud analysis of the microbiota diversity was conducted. $\alpha$-diversity (diversity within samples) and $\beta$-diversity (between 
samples) was evaluated by the OTU (operational taxonomic unit) table. Sobs indices were determined as $\alpha$-diversity and the significance was calculated using Student's $t$-test. Phylogenetic (UniFrac) distance matrices were determined for $\beta$-diversity measurements. The $\mathrm{R}$ package vegan was used to generate community heatmaps and Qiime 1.9 .1 was used to generate cumulative distribution plots of $\beta$-diversity distances. The software PICRUSt 1.0 .0 was used to generate heatmap-based KEGG pathways for the predicted functions.

\subsection{Statistical Analysis}

The SPSS 22.0 software was used to statistical analysis. For normal distributed data, student's $t$-test was performed and for nonnormal data the Wilcoxon signed-rank test was used to compare the two groups. $P<0.05$ was considered statistically significant.

\section{Results}

\subsection{Effects of gestational age on the composition of the gut microbiota}

The composition of the gut microbiota of full-term and preterm in 3-day-old neonates is shown in Figures 1 while 3042-day-old infants is shown in Figures 2. In Figure 1, Escherichia-Shigella, Streptococcus, Bifidobacterium, and Bacteroides were the most abundant genera found in the full-term group, while Staphylococcus, Streptococcus, Escherichia-Shigella and Clostridium were the predominate genera in the preterm group. There was significant difference between the two groups. $(P<0.05)$. As shown in Figure 2, Bifidobacterium, Lactobacillus, Bacteroides, and Clostridium were the main observed genera in the full-term group, whereas Escherichia-Shigella, Clostridium, Bifidobacterium and Bacteroides were more abundant in the preterm group. These differences in microbial composition were statistically significant $(P<0.05)$.

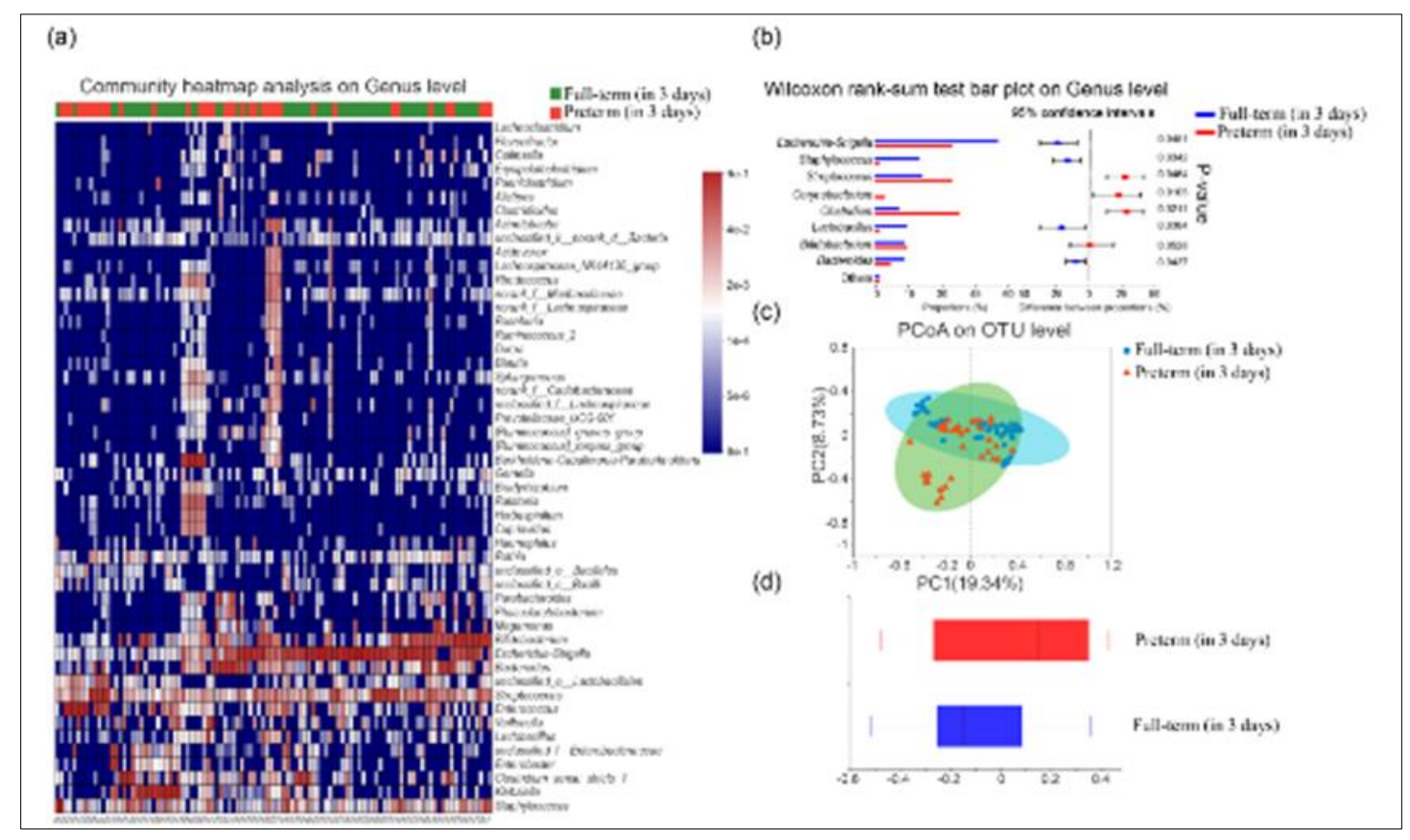

Figure 1 Gut microbiota community composition of two groups (full-term and preterm) in 3-day-old neonates

- (A) Gut microbiota community heatmap analysis of two groups on Genus level. (B) Wilcoxon rank-sum test bar plot between 2 groups on Genus level. (C) Principal coordinate analysis (PCoA) on unweighted UniFrac distances between the neonatal microbiota is shown along the first two principal coordinate (PC) axes. Each point is colored by gestational age and represents a sample: Full-term, blue; Preterm, red. (D) PCoA box diagram. Represents the discrete distribution of two groups of samples on the PC1 axis: Full-term, blue; Preterm, red. 


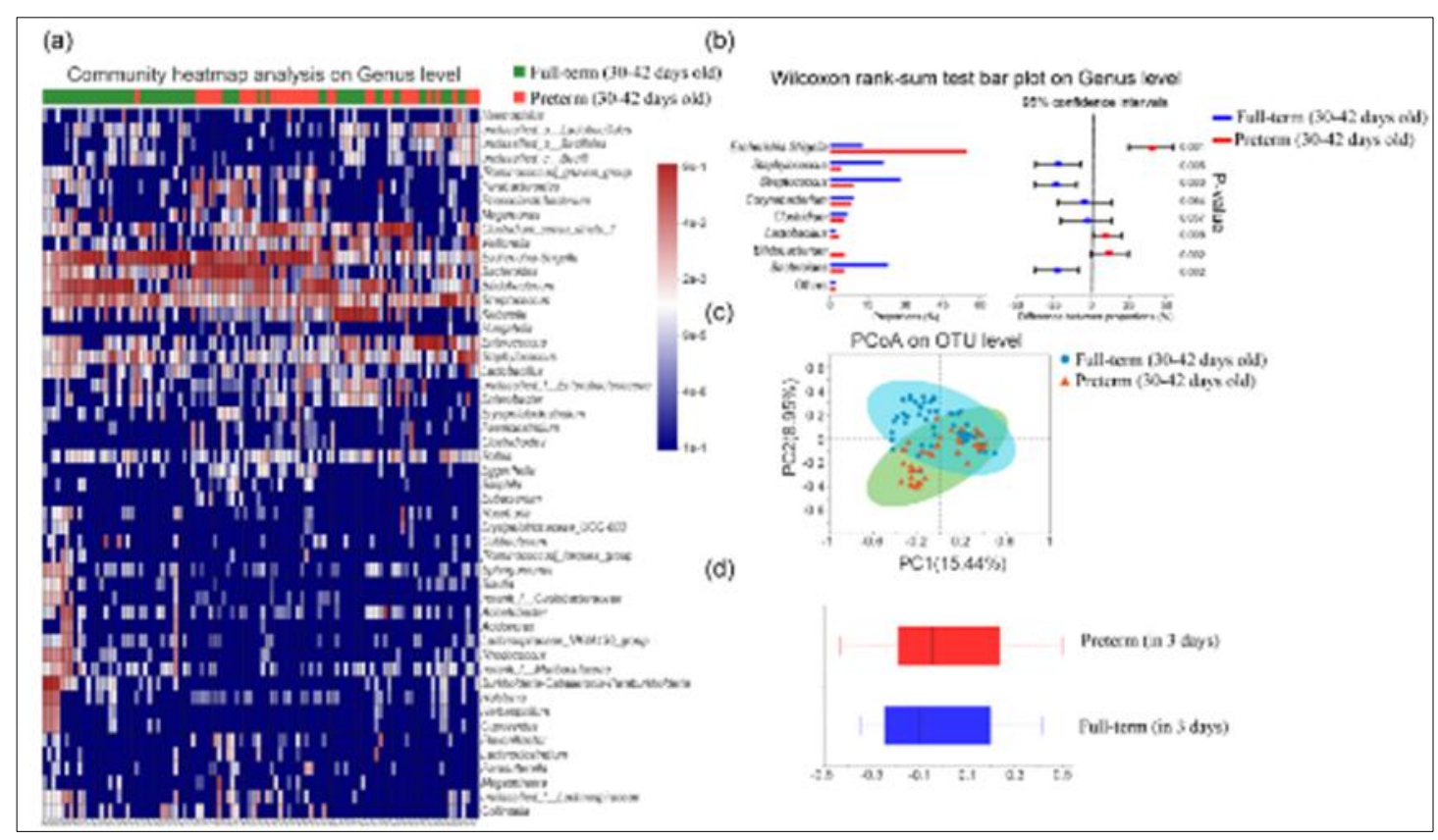

Figure 2 Gut microbiota community composition of 2 groups (full-term and preterm) in 30-42-day-old infants

- (A) Gut microbiota community heatmap analysis of 2 groups on Genus level. (B) Wilcoxon rank-sum test bar plot between 2 groups on Genus level. (C) Principal coordinate analysis (PCoA) on unweighted UniFrac distances between the infants gut microbiota is shown along the first two principal coordinate (PC) axes. Each point is colored by gestational age and represents a sample: Full-term, blue; Preterm, red. (D) PCoA box diagram. Represents the discrete distribution of two groups of samples on the PC1 axis: Full-term, blue; Preterm, red.

\subsection{Sobs indices analysis for $\alpha$ - diversity measurements}

in Figure 3,student's $t$-test was used to calculate the sobs indices of the gut microbiota present in the full-term and preterm groups at days 3- and 30-42 after birth. Although microbiota was detected in all stool samples from 3-day-old neonates, their sobs indices ( $\alpha$-diversity) was significantly lower than that of the 30-42-day-old group, irrespectively of the gestational age $(P<0.001)$.

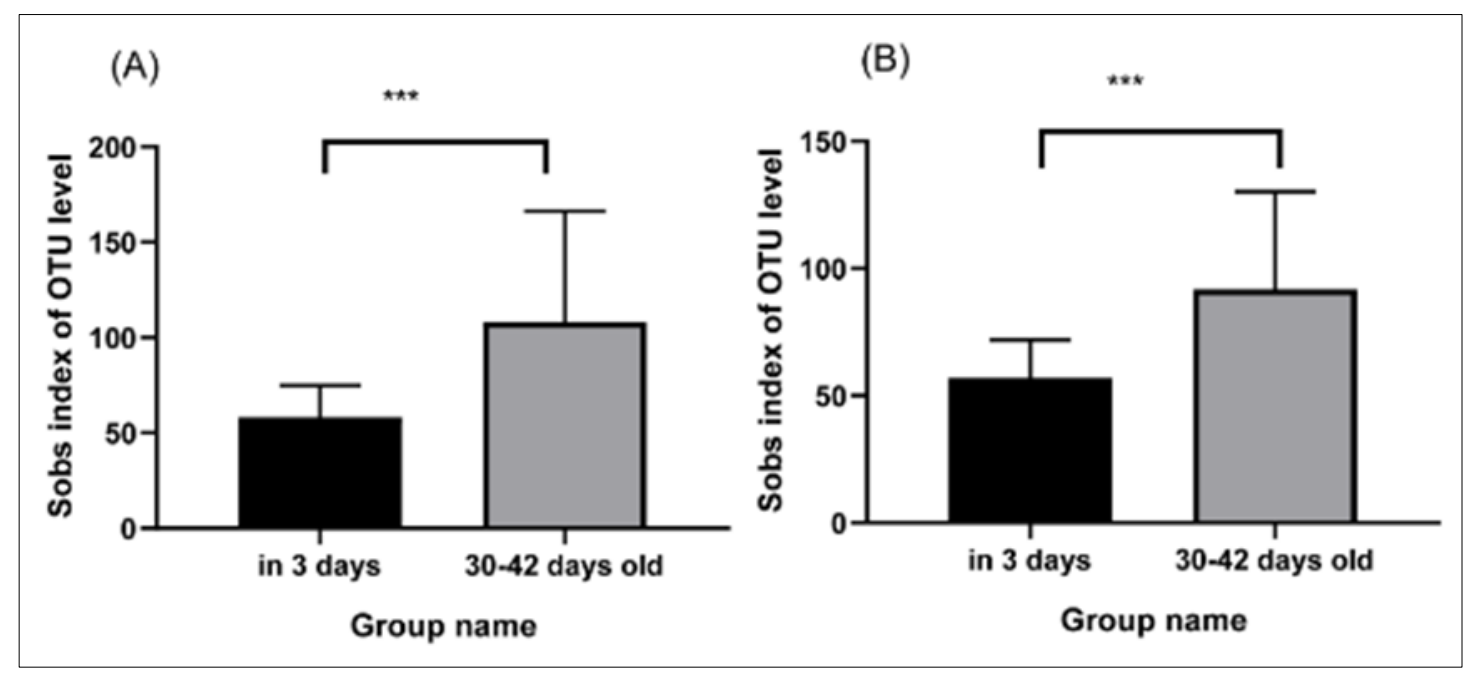

Figure 3 Student's $t$-test for sobs indices of full-term and preterm groups' gut microbiota at 2 points (in 3 days and 30-42-day-old)

- (A) Sobs indices at the 2 points of full-term infants. (B)Sobs indices at the 2 points of preterm infants. 


\subsection{Predicted function of the gut microbiota}

In Figure 4, the predicted function of the gut microbiota of the full-term and preterm groups at days 3- and 30-42 after birth is shown. Heatmap of the Kyoto Encyclopaedia of Genes and Genomes (KEGG) pathway level 2 at 2 points belonging to the categories that showed statistical differences between full-term and preterm groups. Compared to the preterm group, the full-term group of both neonates and infants presented a higher metabolic and biodegradation rates of carbohydrates, vitamins, and xenobiotics, which favours the stability of their microbiota community.

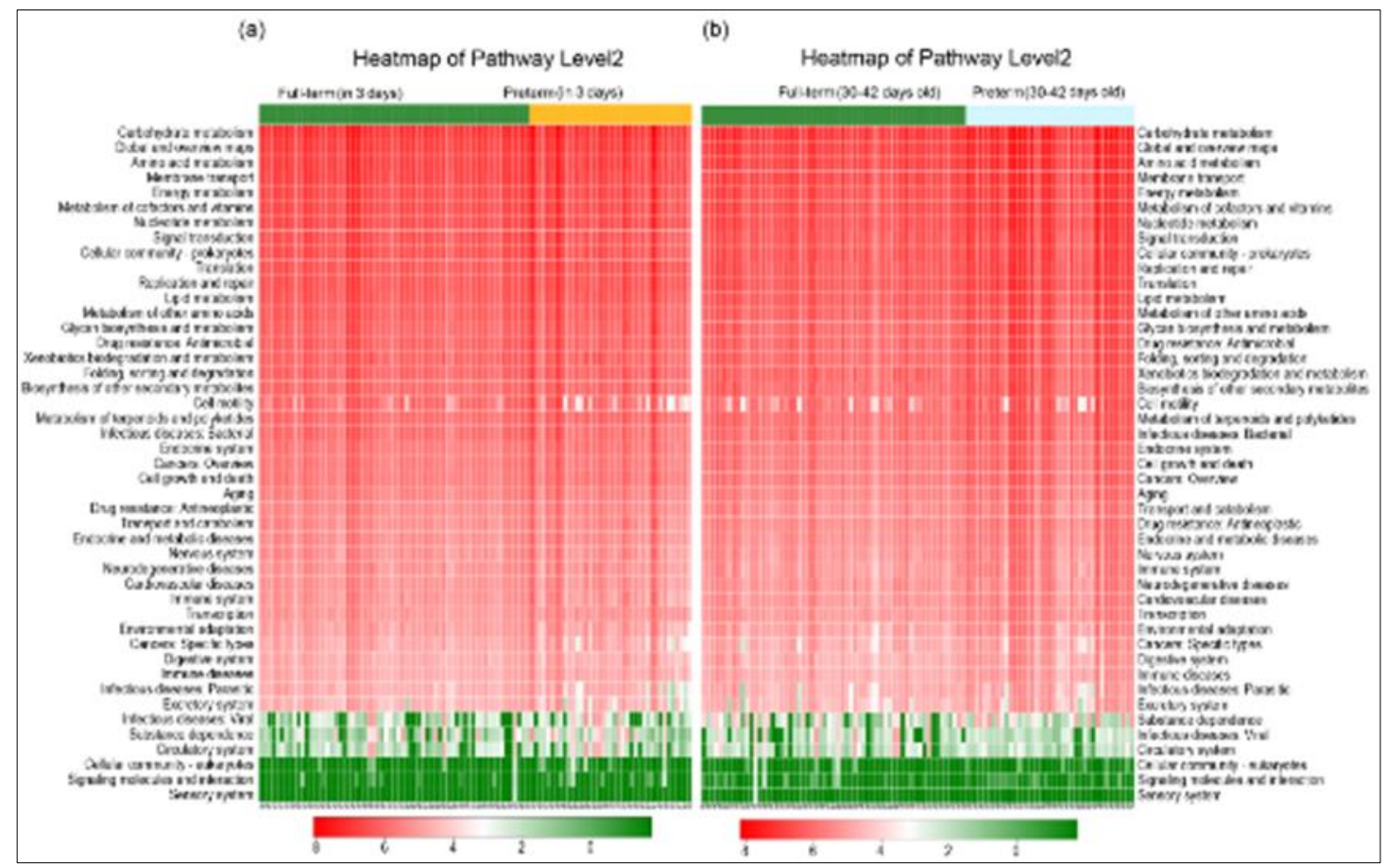

Figure 4 Predicted function of the gut microbiota : Heatmap of KEGG pathway level 2

- (A) Heatmap of KEGG pathway level 2 showing distinct microbial gene profiles of 2 groups' stool in 3 days after birth: Full-term, green; Preterm, black. (B) Heatmap of KEGG pathway level 2 showing distinct microbial gene profiles of 2 groups in 30-42-day-old: Full-term, blue; Preterm, red.

\section{Discussion}

In this study, the levels of beneficial microorganisms such as Bifidobacterium, Lactobacillus, and Bacteroides in full-term group were higher than in preterm, at days 3 and 30-42 after birth. These data were consistent with previous findings by Arboleya $S$ et al. [8]. It has been reported that lactate and acetate, the primary and end metabolic products of the metabolism of Bifidobacterium, a member of the Actinobacteria phylum, are an important energy sources for colonocytes [9]. Moreover, these bacteria can also produce basic nutrients, including riboflavin and folate [10]. Lactobacillus can adjust the normal flora and improve function of the gastrointestinal tract.

In stool samples from 3-day-old neonates, the levels of Staphylococcus were significantly higher in the preterm group than in the full-term, which confirmed the observations previously published on the typical composition of gut microbiota of early infant stools at this age [11]. The levels of Escherichia-Shigella were significantly lower in preterm infants than in full-term, this microbial group was considered derived from maternal stools [12]. The level of Streptococcus and Clostridium were higher in the preterm group than in the full term. In this sense, the presence of Streptococcus in the intestines of infants during early life is usually not harmed as these are human symbiotic bacteria; however, it is important to note that they are also conditional pathogens; and Clostridium is an enteropathogenic bacterium [13]. In the stools from 30-42-day-old infants, the genera Escherichia-Shigella was more abundant in the preterm group than in the full-term. Escherichia-Shigella has been considered by former researcher to be a harbinger of dysbiosis and health harm [14]. This bacterial formation could be explained by the fact that compared to full-term infants, preterm infants were more common born by cesarean section and placed into a highly sanitized intensive care environment, separated from their mother. An endpoint of 30-42 days after delivery was chosen because at this age 
infants have not been exposed to a wide range of environmental sources of microbes due to limited person-to-person contact.

The predicted function analysis found that compared to the preterm group, the full-term group presented higher levels of biodegradation and metabolism of carbohydrates, vitamins which help to the stability of gut microbiota.

It is important to note that the feeding habits of the infants were variable at the end of this study. Therefore, the limitation is that the effect of feeding habits on the results cannot be ruled out between the full-term and preterm groups.

There are nine hypervariable specificity regions in the bacterial $16 \mathrm{~S}$ rRNA gene that vary depending on the genus or species. Therefore, 16S rRNA is considered the most important indicator for bacterial phylogenetic, and strain identification, even at the species level [15]. 16S rRNA Amplicon Sequencing analysis and taxonomic identification are important means for researching microbiota composition in environment samples [16]. At present, 16S sequencing technology is gradually being developed in China. For example, Nanjing' Jinling Hospital used 16S sequencing technology to detect constipation in a mouse model; and Shanghai Jiao Tong University used 16S sequencing technology to detect a gut microbiota-targeted dietary intervention [17] [18]. In this study, we used 16S sequencing technology to characterize the gut microbiota in early infants and provided scientific and accurate data resources for the establishment of an early infant gut microbiota databank.

Overall, our results indicated that the capacity of the gut of preterm infants to establish a normal microbiota, in terms of composition and function, is lower than that of full-term infants; this deficiency was observed at both time points studied. Due to the important influence of the microbiota during early life on the maturation of the immune system, this inability may produce a risk for their future health. We believe that routine treatment of preterm infants with probiotics could help with the establishment of a healthy microbiota.

\section{Conclusion}

There were significant differences in the composition and predicted function of the gut microbiota and the level of probiotics in early infants owing to gestational age. 16S sequencing technology is reliable and effective in the detection of gut microbiota in early infants.

\section{Compliance with ethical standards}

\section{Acknowledgments}

We would like to thank Editage (www.editage.cn) for English language editing.

\section{Disclosure of conflict of interest}

There are no conflicts of interest to declare.

\section{Statement of informed consent}

Informed consent was obtained from all individual participants included in the study.

\section{Data Availability}

All data relevant to the study are included in the article. Raw faecal samples and bacterial isolates are available from the corresponding authors upon request.

\section{Funding Statement}

This research is supported by Hangzhou science and technology plan guidance project in Hangzhou city (Number: 20181228Y81). Moreover, it is supported by Hangzhou Medical and health science and technology project (Number: 0020190871). 


\section{References}

[1] S Matamoros, C Gras-Leguen, F Le Vacon, G Potel, MF de La Cochetiere. Development of gut microbiota in infants and its impact on health, Trends Microbiol. 2013; 21: 167-173.

[2] K Sim, E Powell, AG Shaw, Z McClure, M Bangham, JS Kroll. The neonatal gastrogut microbiota: the foundation of future health?, Arch Dis Child Fetal Neonatal Ed. 2013; 98: F362-364.

[3] MJ Blaser, S Falkow. What are the consequences of the disappearing human microbiota?, Nat Rev Microbiol. 2009; 7: 887-894.

[4] EK Costello, K Stagaman, L Dethlefsen, BJ Bohannan, DA Relman. The application of ecological theory toward an understanding of the human microbiome, Science. 2012; 336: 1255-1262.

[5] LA David, CF Maurice, RN Carmody, DB Gootenberg, JE Button, BE Wolfe, AV Ling, AS Devlin, Y Varma, MA Fischbach, SB Biddinger, RJ Dutton, PJ Turnbaugh. Diet rapidly and reproducibly alters the human gut microbiome, Nature. 2014; 505: 559-563.

[6] O Koren, JK Goodrich, TC Cullender, A Spor, K Laitinen, HK Backhed, A Gonzalez, JJ Werner, LT Angenent, R Knight, F Backhed, E Isolauri, S Salminen, RE Ley. Host remodeling of the gut microbiome and metabolic changes during pregnancy, Cell. 2012; 150: 470-480.

[7] M Reyman, MA van Houten, D van Baarle, A Bosch, WH Man, M Chu, K Arp, RL Watson, EAM Sanders, S Fuentes, D Bogaert. Impact of delivery mode-associated gut microbiota dynamics on health in the first year of life, Nat Commun. 2019; 10: 4997.

[8] S Arboleya, B Sanchez, G Solis, N Fernandez, M Suarez, AM Hernandez-Barranco, C Milani, A Margolles, CG de Los Reyes-Gavilan, M Ventura, M Gueimonde. Impact of Prematurity and Perinatal Antibiotics on the Developing Gut Microbiota: A Functional Inference Study, Int J Mol Sci. 2016; 17.

[9] S Fukuda, H Toh, K Hase, K Oshima, Y Nakanishi, K Yoshimura, T Tobe, JM Clarke, DL Topping, T Suzuki, TD Taylor, K Itoh, J Kikuchi, H Morita, M Hattori, H Ohno. Bifidobacteria can protect from enteropathogenic infection through production of acetate, Nature. 2011; 469: 543-547.

[10] H Sugahara, T Odamaki, N Hashikura, F Abe, JZ Xiao. Differences in folate production by bifidobacteria of different origins, Biosci Microbiota Food Health. 2015; 34: 87-93.

[11] MJ Butel, AJ Waligora-Dupriet, S Wydau-Dematteis. The developing gut microbiota and its consequences for health, J Dev Orig Health Dis. 2018; 9: 590-597.

[12] DM Chu, J Ma, AL Prince, KM Antony, MD Seferovic, KM Aagaard. Maturation of the infant microbiome community composition and function across multiple body sites and in relation to mode of delivery, Nat Med. 2017; 23: 314326.

[13] A Partty, R Luoto, M Kalliomaki, S Salminen, E Isolauri. Effects of early prebiotic and probiotic supplementation on development of gut microbiota and fussing and crying in preterm infants: a randomized, double-blind, placebo-controlled trial, J Pediatr. 2013; 163: 1272-1277 e1271-1272.

[14] NR Shin, TW Whon, JW Bae. Proteobacteria: microbial signature of dysbiosis in gut microbiota, Trends Biotechnol. 2015; 33: 496-503.

[15] JG Caporaso, CL Lauber, WA Walters, D Berg-Lyons, CA Lozupone, PJ Turnbaugh, N Fierer, R Knight. Global patterns of $16 \mathrm{~S}$ rRNA diversity at a depth of millions of sequences per sample, Proc Natl Acad Sci U S A, 108 Suppl 1. $2011 ; 4516-4522$.

[16] N Youssef, CS Sheik, LR Krumholz, FZ Najar, BA Roe, MS Elshahed. Comparison of species richness estimates obtained using nearly complete fragments and simulated pyrosequencing-generated fragments in 16S rRNA gene-based environmental surveys, Appl Environ Microbiol. 2009; 75: 5227-5236.

[17] X Ge, W Zhao, C Ding, H Tian, L Xu, H Wang, L Ni, J Jiang, J Gong, W Zhu, M Zhu, N Li, Potential role of fecal microbiota from patients with slow transit constipation in the regulation of gastrogut motility, Sci Rep. 2017; 7 : 441.

[18] S Xiao, N Fei, X Pang, J Shen, L Wang, B Zhang, M Zhang, X Zhang, C Zhang, M Li, L Sun, Z Xue, J Wang, J Feng, F Yan, $\mathrm{N}$ Zhao, J Liu, W Long, L Zhao. A gut microbiota-targeted dietary intervention for amelioration of chronic inflammation underlying metabolic syndrome, FEMS Microbiol Ecol. 2014; 87: 357-367. 